\title{
La severidad de la artritis reumatoide como predictor oportuno de inestabilidad de la columna cervical asintomática
}

\author{
Geraldo-Flores NA,* Merlos-López RJ,** Rodríguez-Wong JA, *** Ramírez-Hernández S,**** \\ Espino-Lizarraga MJ,***** Pérez-Atanasio JM******
}

Hospital Regional 2. IMSS Villa Coapa

RESUMEN. Introducción: La afectación de la columna cervical es común en pacientes con artritis reumatoide (AR), factores de riesgo como la actividad de la enfermedad puede estar relacionada con inestabilidad cervical asintomática. Objetivo: Determinar los factores asociados a la inestabilidad de la columna cervical asintomáticos en pacientes con artritis reumatoide. Material y métodos: Estudio de casos y controles provenientes de la consulta externa de reumatología y columna de un centro de trauma de nivel II para identificar a todos los pacientes diagnosticados con AR e inestabilidad cervical asintomáticos. Con radiografías simples de la columna cervical que hacen mediciones radiográficas, se realizó el diagnóstico de inestabilidad cervical, se evaluaron los factores de riesgo como la presencia de factor reumatoide (FR), cirugías articulares previas, las elevaciones de los valores de proteína $\mathrm{C}$ reactiva (PCR), la severidad y actividad de la enfermedad medida en el índice de actividad de la enfermedad de $\mathbf{2 8}$ articulaciones (DAS 28), además del índice de actividad de la enfermedad simplificada (SDAI). Resultados: Se evaluaron 32 pacientes, nueve $(\mathbf{2 8 . 1} \%)$ cumplieron con los criterios para inestabilidad de la colum-
ABSTRACT. Introduction: Cervical spine involvement is common in patients with $R A$, risk factors such as disease activity may be related to asymptomatic cervical instability. Objective: To determine the associated factors for asymptomatic cervical spine instability in patients with rheumatoid arthritis. Material and methods: Case and control study from the external spine and rheumatology consultation of a level II trauma center to identify all patients diagnosed with rheumatoid arthritis (RA) and asymptomatic cervical instability. With simple X-rays of the cervical spine, carrying out radiographic measurements, the diagnosis of cervical instability was performed, risk factors such as the presence of rheumatoid factor (FR), previous articular surgeries, elevations of the C-reactive protein (PCR) values were evaluated. The severity and activity of the disease measured in the activity index of 28 articulations (DAS 28 ) in addition to the index of activity of the simplified disease (SDAI). Results: We assessed 32 patients, nine patients $(\mathbf{2 8 . 1} \%)$ met the criteria for instability of the anterior cervical spine atlantoaxial subluxation (SAAa) $(\mathbf{1 0 0 \%})$, also one

Nivel de evidencia: IV

\footnotetext{
* Ortopedista. Hospital Regional 2. IMSS Villa Coapa.

** Reumatología. Hospital Regional 2. IMSS Villa Coapa.

*** Ortopedista. Jefe del Servicio de Columna. Hospital Regional 2. IMSS Villa Coapa.

**** Ortopedista. Servicio de Columna. Hospital Regional 2. IMSS Villa Coapa.

***** Licenciada en Nutrición. Universidad del Valle de México Campus Coyoacán, Ciudad de México.

****** Ortopedista, Maestría en Ciencias Médicas. Hospital Regional 2. IMSS Villa Coapa.

Dirección para correspondencia:

Dr. José Manuel Pérez-Atanasio

Periférico Sur Núm. 7650, 30-402, Granjas Coapa, CP 14330, Tlalpan, Ciudad de México.

Cel: 5534143186

E-mail: drmanuelperezata@gmail.com
}

Este artículo puede ser consultado en versión completa en http://www.medigraphic.com/actaortopedica 
na cervical con subluxación atlantoaxial anterior (SAAa) $(\mathbf{1 0 0 \%})$, también un paciente con SAAa presentaba subluxación vertical (SV), los factores de riesgo más relevantes: el DAS 28 con un $\mathrm{OR}=$ 3.54, SDA con un $\mathrm{OR}=\mathbf{2 . 3 4}$ y por último el $\mathrm{PCR}>$ 1.0 su $\mathrm{OR}=2.88$. Conclusión: Los factores de riesgo asociados oportunamente en nuestra población son la severidad de la actividad de la enfermedad que podemos observar en el DAS y SDAI al aplicarlos en los pacientes y PCR $>$ 1.0.

Palabras clave: Columna cervical, inestabilidad, artritis reumatoide, articulación atlantoaxial, factores de riesgo.

\section{Introducción}

Los procesos inflamatorios en la artritis reumatoide (AR) afectan principalmente las articulaciones de manos y pies y en tercer lugar se encuentra la columna cervical. ${ }^{1}$

La inestabilidad de la columna cervical en pacientes con AR tiene una incidencia entre $9-88 \%$ y se caracteriza por ser progresiva. ${ }^{2}$ Las lesiones inflamatorias y erosivas de la columna cervical con subluxación e inestabilidad se presentan tempranamente, a menudo dentro de los primeros dos a 10 años después del diagnóstico de la AR. ${ }^{2,3}$

En la columna cervical superior el tipo más común de inestabilidad entre las vértebras $\mathrm{C} 1$ y $\mathrm{C} 2$ es la subluxación atlantoaxial (SAA), la prevalencia oscila entre 19 y $70 \%$ debido al debilitamiento o ruptura de los ligamentos de la articulación atlantoaxial, así como a la ulceración del hueso subcondral. La subluxación atlantoaxial anterior (SAAa) ocurre con mayor frecuencia aproximadamente en $75 \%$ de todas las SAA, la posterior y lateral no son frecuentes. $20 \%$ de los pacientes presentan subluxación vertical (SV) con lesión de las vértebras C3 a C7, otra patología es subluxación subaxial (SS) resultado de la migración superior de la odontoides al foramen magnum en $15 \%$ de los pacientes. ${ }^{1,3,4}$

Las manifestaciones clínicas de las AR en la columna cervical pueden ser bastante variables, la mayoría son asintomáticas durante años. Cincuenta por ciento muestra progresión radiológica durante su seguimiento, en ocasiones la clínica no se correlaciona con el aspecto radiográfico. En adultos menores de 40 años asintomáticos, 25\% presenta evidencia radiográfica de inestabilidad cervical, aumentando a $50 \%$ en adultos mayores de 40 años y alcanzando $85 \%$ en mayores de 60 años. ${ }^{5,6}$

Un tercio de los pacientes con subluxación de columna cervical son asintomáticos, por lo tanto, este estado clínico deberá ser considerado por los cirujanos de columna, reumatólogos y cualquier personal de salud para una detección oportuna y prevenir complicaciones graves, incluyendo tetraplejía, compresión medular y muerte súbita. ${ }^{7}$ patient with SAAa presented vertical subluxation (SV), risk factors more relevant: DAS 28 with an $\mathrm{OR}=\mathbf{3 . 5 4}$, $\mathrm{SDA}$ with an $\mathrm{OR}=\mathbf{2 . 3 4}$ and finally the $\mathrm{PCR}>1.0$ its $\mathrm{OR}=2.88$. Conclusion: The risk factors associated opportunely in our population are the severity of the activity of the disease that we can see in the DAS and SDAI when applied in patients and $\mathrm{PCR}>\mathbf{1 . 0}$.

Key words: Cervical spine, instability, rheumatoid arthritis, atlantic-axial articulation, risk factors.
Se han reportado en la literatura varios factores de riesgo: sexo (femenino), edad al diagnóstico de AR, duración de la enfermedad, erosión en las articulaciones periféricas (mano y pie), presencia de factor reumatoide (FR), antecedente de cirugías articulares previas, elevación de los valores de proteína $\mathrm{C}$ reactiva (PCR), velocidad de sedimentación globular (VSG), severidad y actividad de la enfermedad de acuerdo con el índice de actividad de la enfermedad de 28 articulaciones (DAS 28) y por el índice de actividad de la enfermedad simplificada (SDAI). Los factores de riesgo y prevalencia varían sobre los temas estudiados y los criterios diagnósticos. ${ }^{2,8,9}$

El reconocimiento precoz de estos factores y condiciones clínicas permiten un tratamiento oportuno para pacientes con indicaciones de tratamiento quirúrgico, asociándose tasas más bajas de complicaciones y mejores resultados funcionales. ${ }^{6}$

El presente estudio tuvo como objetivo evaluar los factores de riesgo de inestabilidad de la columna cervical asintomáticos en pacientes con AR. De acuerdo a la literatura revisada existen pocos estudios en este tipo de pacientes.

\section{Material y métodos}

Seleccionamos pacientes con AR en consulta externa del servicio de reumatología y columna de un hospital de segundo nivel, fueron reclutados de Noviembre de 2016 a Octubre de 2017. Todos los pacientes cumplieron con los criterios de la Liga Europea Contra el Reumatismo (EULAR) para la AR. Se evaluó un total de 32 pacientes, resultando nueve con afección cervical asintomáticos. Se realizó un estudio de casos y control. De los registros médicos se obtuvieron los datos de género, edad, duración de la enfermedad, historial de medicamentos, antecedentes de cirugías articulares, como sinovectomía artroscópica o reemplazo articular; aplicación de escalas para valorar la severidad y actividad de la AR (DAS 28, SDAI). Referente a laboratorio los datos para este análisis incluyeron VSG, PCR y VSG. Las radiografías de columna cervical que se incluyeron fueron: lateral neutra, lateral en flexión, en extensión y de boca 
abierta. La SAA anterior se midió registrando la distancia entre la superficie posterior del arco anterior del atlas y la superficie anterior del proceso odontoide. Se consideró que la separación de $2.5 \mathrm{~mm}$ era significativa. La SV se diagnosticó cuando la punta de la odontoide se encontraba a más de $4.5 \mathrm{~mm}$ por encima de la línea descrita por McGregor (una línea dibujada entre el paladar duro y el punto más caudal de la curva occipital). La SS se diagnosticó cuando una vértebra estaba más de $2 \mathrm{~mm}$ en relación con la próxima vértebra, se midió desde la línea posterior de la columna vertebral. La presencia de erosiones fue revelada por las radiografías de las manos en proyección posteroanterior. Análisis estadístico. Se compararon las distribuciones de variables continuas entre grupos realizando prueba $t$ de Student, para comparar variables categóricas se aplicó la $\chi^{2}$. El valor $\mathrm{p}<0.05$ se consideró significativo. La determinación de riesgo se realizó obteniendo el OR en un análisis multivariado, considerando casos de pacientes que presentaron inestabilidad de la columna cervical asintomática. Todos los análisis fueron realizados utilizando SPSS v. 20 (IBM Corp., Armonk, NY, EUA).

\section{Resultados}

Se evaluaron 32 pacientes con AR asintomáticos, de sexo masculino fueron tres $(9.37 \%)$ y femenino $29(90.62 \%)$ con una edad media de $47.6 \pm 9.9$ años. El tiempo de diagnóstico de AR en estos pacientes resultó una media de $112.88 \pm$ 69.89 meses (Tabla 1).

Se evaluaron estudios radiográficos simples de la columna cervical, los cuales revelaron que nueve $(28.1 \%)$ presen- taban inestabilidad cervical sin sintomatología neurológica y/o dolor en cuello, tres (33.33\%) del sexo masculino y seis (66.7\%) femenino.

El tipo de inestabilidad de la columna cervical fue SAA en los nueve pacientes $(100 \%)$, tenían distancia anterior atlantoaxial entre 2.5 y $5.0 \mathrm{~mm}$; además, uno (11.1\%) presentaba SV y SAA.

Al comparar los pacientes con inestabilidad de la columna cervical con quienes no tenían dicha inestabilidad, fue más frecuente en el sexo femenino. El uso de medicamentos como terapia combinada con fármacos antirreumáticos modificadores de la enfermedad (FARME) y terapia biológica fue más frecuente en pacientes con afección de la columna cervical. Los pacientes con AR con inestabilidad de la columna cervical eran más jóvenes que los que no tenían afectación (43.1 vs. 48.8 años, $p=0.143$ ); los valores elevados del factor reumatoide y la historia de cirugía articular son más frecuentes en pacientes con afectación de la columna cervical que en los pacientes sin afección (148.44 vs. 94.60, 11.1 vs. $0 \%$ ) de la misma forma, los valores elevados de VSG y PCR (31.3 vs. $29.95,1.96$ vs. 1.00$)$.

Usando un análisis multivariado, los factores de riesgo de inestabilidad de la columna fueron la severidad de la enfermedad de la AR medida con el DAS $28(\mathrm{OR}=3.54, \mathrm{p}=$ $0.123)$ y SDAI $(\mathrm{OR}=2.34, \mathrm{p}=0.287)$ además del PCR > $1.0(\mathrm{OR}=2.88, \mathrm{p}=0.207)($ Tabla 2$)$.

\section{Discusión}

En este estudio, lo primero fue evaluar los factores de riesgo que afectan la columna cervical en pacientes con $\mathrm{AR}$

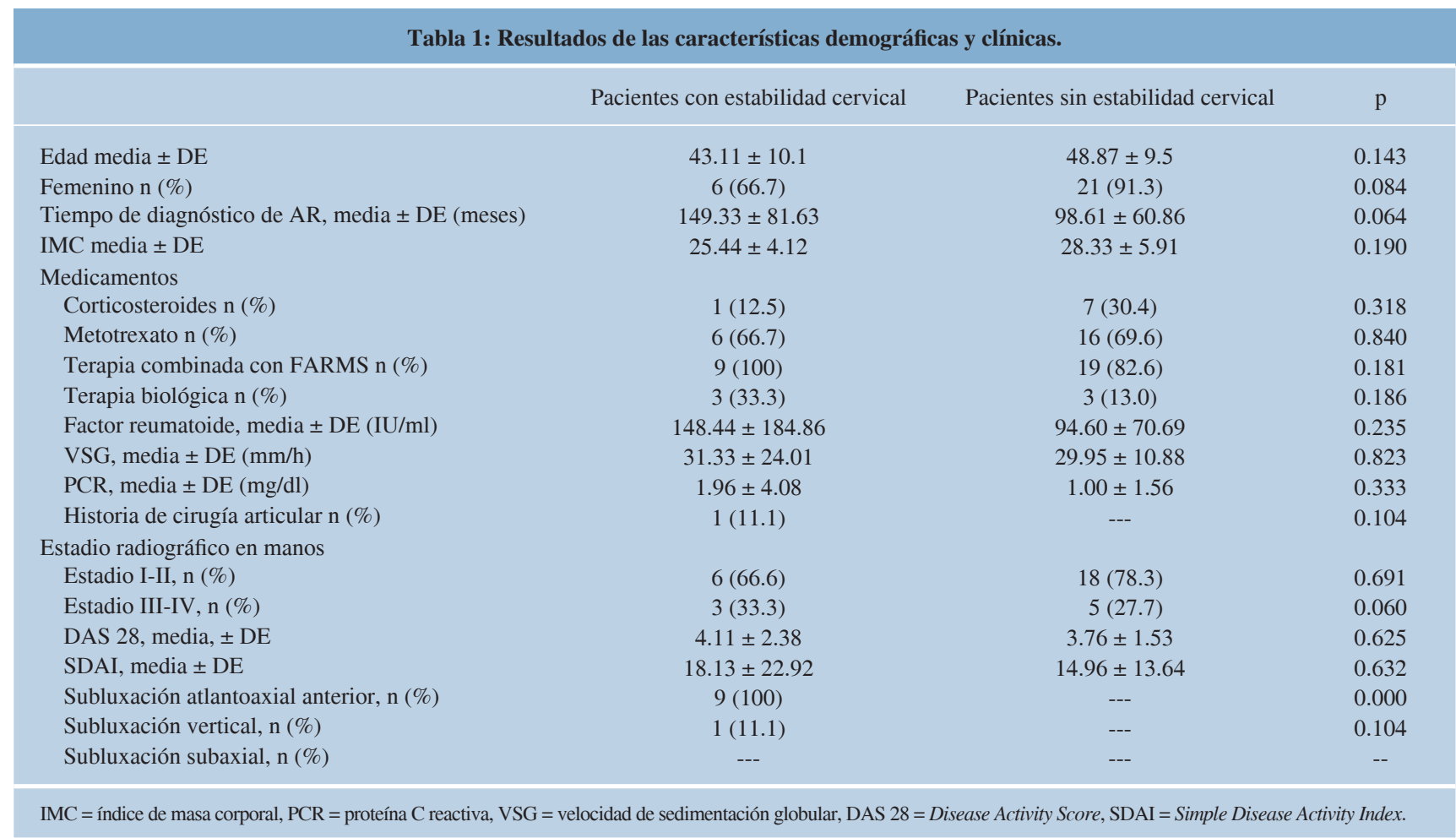




\begin{tabular}{|c|c|c|c|}
\hline Variables independientes & OR & $95 \%$ IC & $\mathrm{p}$ \\
\hline DAS 28 & 3.54 & $0.70-17.73$ & 0.123 \\
\hline SDAI & 2.34 & $0.48-11.26$ & 0.287 \\
\hline Estadio radiográfico & 0.80 & $0.13-4.74$ & 9.805 \\
\hline Tiempo de diagnóstico de AR & 0.43 & $0.11-1.59$ & 0.306 \\
\hline $\mathrm{VSG}>40$ & 1.80 & $0.32-9.88$ & 0.498 \\
\hline $\mathrm{PCR}>1.0$ & 2.88 & $0.55-14.94$ & 0.207 \\
\hline EDAD $>45$ años & 0.44 & $0.08-2.20$ & 0.319 \\
\hline Mujer & 0.19 & $0.02-1.41$ & 0.105 \\
\hline Factor reumatoide & 0.80 & $0.16-3.81$ & 0.783 \\
\hline IMC sobrepeso & 1.06 & $0.20-5.44$ & 0.938 \\
\hline
\end{tabular}

que presentan inestabilidad de la columna cervical. Una de nuestras principales observaciones fue que de los 32 pacientes evaluados con AR, ninguno presentaba sintomatología. Teniendo en cuenta la frecuencia de afectación de la columna vertebral en pacientes con AR asintomáticos, la verdadera prevalencia puede estar subestimada porque no se realizan protocolos en estos pacientes para una detección oportuna. Estos hallazgos indican la necesidad de monitorear esta condición para prevenir sus complicaciones graves.

La SAA fue el hallazgo más común con un cambio de la distancia atlantoaxial anterior entre 2.5-5.0 mm y en segundo lugar el tipo de inestabilidad cervical resultó la SV.

En muchos estudios se han buscado factores de riesgo que afectan la columna cervical en pacientes con AR y los factores de riesgo comúnmente informados incluyen erosiones de las articulaciones periféricas, FR, edad avanzada, sexo masculino y la enfermedad activa. Nuestro estudio también encontró asociaciones entre PCR elevada y la severidad de la enfermedad activa; nuestros hallazgos apoyan estudios que identificaron el estado de enfermedad activa como predictor de SAA. Estos hallazgos indican una inflamación activa y destructiva de las articulaciones en pacientes con afectación cervical reumatoide. ${ }^{8}$

Otros estudios han identificado factores de progresión de la enfermedad cervical, incluidos los de género, la seropositividad del factor reumatoide, la enfermedad, el uso prolongado de corticosteroides, altos niveles de PCR. ${ }^{5}$ Estos estudios revelaron frecuentemente hallazgos cervicales definidos radiológicamente y deterioro de la columna vertebral en sus poblaciones de estudio que se encuentran correlacionados con la progresión de las lesiones cervicales como lo reportado en nuestro estudio.

Collin et al. mencionan que la prevalencia de la subluxación de la columna cervical asintomática en pacientes con AR es alta, 49\% y de $38 \%$ para SV y SV. ${ }^{10}$

Recientemente, Mohammadali Nazarinia et al. señalan en su estudio que de 100 pacientes, 17 (17\%) presentaban subluxación de la columna cervical asintomática. ${ }^{11}$

Otro estudio realizado por Kauppi MJ et al. demostró que los pacientes con AR con clínica sostenida de la actividad de la enfermedad tienen mayor riesgo de desarrollar SAAa. ${ }^{12}$
En un estudio realizado por Yi Liu et al. los resultados indicaron que los niveles más altos de VSG, PCR y DAS 28 estaban significativamente correlacionados con afección de la columna cervical en la AR, los cuales constituyen un importante factor de riesgo de inestabilidad cervical en pacientes con AR. También Takashi Kaito et al. mencionan que están relacionados con la discapacidad física y la destrucción articular radiográfica y son predictores precisos de la progresión como en nuestro estudio. , $^{8,10,11}$

Un alto valor de DAS-PCR antes del inicio puede estar relacionado con la sinovitis y la destrucción en las extremidades y en las articulaciones cervicales atlantoaxial y facetarías. Estos indicadores de actividad reflejaron únicamente un cierto punto del tiempo en el largo curso de la AR. Estas asociaciones de factores de riesgo requieren un estudio de seguimiento a largo plazo. ${ }^{2}$

El presente estudio puede generar críticas por el tamaño pequeño de la muestra y el tiempo de seguimiento de los pacientes; se necesitarán estudios adicionales para valorar a los pacientes con inestabilidad de la columna cervical asintomáticos con AR para poder realizar un protocolo de estudio para la detección y manejo oportuno con miras a una mejor calidad de vida y reducción de complicaciones. ${ }^{13}$

\section{Conclusión}

Nuestra población de pacientes con AR suele mostrar afección de la columna cervical asintomática y debe ser tratada por un equipo multidisciplinario de médicos familiares, reumatólogos y ortopedistas. Los factores de riesgo asintomáticos asociados a la afectación de la inestabilidad de la columna cervical incluyen los valores de PCR $>1.0$ y la severidad de la actividad de la enfermedad evaluada con el DAS 28 y SDAI para su detección oportuna.

Bibliografía

1. Mańczak M, Gasik R. Cervical spine instability in the course of rheumatoid arthritis - imaging methods. Reumatologia. 2017; 55(4): 201-7.

2. Kaito T, Ohshima S, Fujiwara H, Makino T, Yonenobu K. Predictors for the progression of cervical lesion in rheumatoid arthritis under the treatment of biological agents. Spine (Phila Pa 1976). 2013; 38(26): 2258-63.

3. Del Grande M, Del Grande F, Carrino J, Bingham CO 3rd, Louie GH. Cervical spine involvement early in the course of rheumatoid arthritis. Semin Arthritis Rheum. 2014; 43(6): 738-44.

4. Kaito T, Hosono N, Ohshima S, Ohwaki H, Takenaka S, Fujiwara H, et al. Effect of biological agents on cervical spine lesions in rheumatoid arthritis. Spine (Phila Pa 1976). 2012; 37(20): 1742-6.

5. Na MK, Chun HJ, Bak KH, Yi HJ, Ryu JI, Han MH. Risk factors for the development and progression of atlantoaxial subluxation in surgically treated rheumatoid arthritis patients, considering the time interval between rheumatoid arthritis diagnosis and surgery. $J$ Korean Neurosurg Soc. 2016; 59(6): 590-6.

6. Cha TD, An HS. Cervical spine manifestations in patients with inflammatory arthritides. Nat Rev Rheumatol. 2013; 9(7): 423-32.

7. Terashima Y, Yurube T, Hirata H, Sugiyama D, Sumi M; Hyogo Organization of Spinal Disorders. Predictive risk factors of cervical spine instabilities in rheumatoid arthritis: a prospective multicenter over 10-year cohort study. Spine (Phila Pa 1976). 2017; 42(8): 556-64. 
8. Zhu S, Xu W, Luo Y, Zhao Y, Liu Y. Cervical spine involvement risk factors in rheumatoid arthritis: a meta-analysis. Int $J$ Rheum Dis. 2017; 20(5): 541-9.

9. Han MH, Ryu JI, Kim CH, Kim JM, Cheong JH, Bak KH, et al. Factors that predict risk of cervical instability in rheumatoid arthritis patients. Spine (Phila Pa 1976). 2017; 42(13): 966-73.

10. Collins DN, Barnes CL, FitzRandolph RL. Cervical spine instability in rheumatoid patients having total hip or knee arthroplasty. Clin Orthop Relat Res. 1991; (272): 127-35.
11. Nazarinia M, Jalli R, Kamali-Sarvestani E, Farahangiz S, Ataollahi M. Asymptomatic atlantoaxial subluxation in rheumatoid arthritis. Acta Med Iran. 2014; 52(6): 462-6.

12. Neva MH, Häkkinen A, Mäkinen H, Hannonen P, Kauppi M, Sokka T. High prevalence of asymptomatic cervical spine subluxation in patients with rheumatoid arthritis waiting for orthopaedic surgery. Ann Rheum Dis. 2006; 65(7): 884-8.

13. Zhang T, Pope J. Cervical spine involvement in rheumatoid arthritis over time: results from a meta-analysis. Arthritis Res Ther. 2015; 17: 148. 